\title{
Prognostic implications of vitamin D in patients with COVID-19
}

\author{
Tom Hosack $^{1}$ - Vadir Baktash ${ }^{1}$ - Amit K. J. Mandal ${ }^{1}$ - Constantinos G. Missouris ${ }^{1,2}$
}

Published online: 23 November 2020

(c) Springer-Verlag GmbH Germany, part of Springer Nature 2020

\section{To The Editor,}

We congratulate Hastie et al. on their short communication [1]. Their study was published soon after the recent rapid evidence review by The National Institute for Health and Clinical Excellence (NICE) [2], supporting the conclusion that there is no substantial evidence to date of an association between vitamin $\mathrm{D}$ deficiency and severity of or mortality from COVID-19. We would like to take this opportunity to report our experience which identified contrasting conclusions to the above.

We recently conducted a prospective cohort study in the United Kingdom (UK) to assess the role of serum vitamin D (25-OH-D) levels in older patients with COVID-19 [3]. Importantly, our study showed that patients with low concentrations of $25 \mathrm{OH}-\mathrm{D}(\leq 30 \mathrm{nmol} / \mathrm{L})$ demonstrated clinically significant, elevated markers of cytokine storm resulting in critical hypoxia requiring non-invasive ventilatory support. Although our study was underpowered to detect a significant mortality difference between the vitamin $\mathrm{D}$ deficient and replete groups, our findings support that Vitamin D status may be a surrogate prognosticator for morbidity and mortality in patients with COVID-19.

There are a few possibilities as to why our results differed from that of Hastie and colleagues. Unlike our study, Hastie et al. obtained a large study sample using historical data from the UK biobank database. Considering evidence of vitamin D possibly being a negative acute phase

A reply to this letter can be read here: doi:https://doi.org/10.1007/ s00394-020-02430-x.

This letter refers to the original publication available here: doi:https://doi.org/10.1007/s00394-020-02372-4.

\section{Constantinos G. Missouris}

dinos.missouris@nhs.net

1 Departments of Cardiology and Internal Medicine, Wexham Park Hospital, Frimley Health NHS Foundation Trust, Wexham Street, Slough, UK

2 University of Cyprus Medical School, Nicosia, Cyprus reactant $[4,5]$, historical data may have been used to help avert from bias secondary to reverse causality. Nonetheless, using historical data from 10 years ago remains a significant limitation to the study. The authors justify this by identifying a subgroup of 15,473 patients that remained vitamin D deficient at follow-up on average 4.3 years later and would therefore have likely remained deficient during the COVID19 pandemic. The major drawback to this inference is the launch of new guidelines on vitamin D supplementation by the National Health Service (NHS) in the UK in July 2016, recommending that everyone should take vitamin D supplementation during winter months [3]. This national effort to tackle long-term mineral and bone disorders after the study's follow-up period is likely to have introduced significant variability in vitamin D statuses of their study population. Multivariate analyses were undertaken at baseline; nonetheless, variables such as smoking status, BMI, comorbidities and socio-demographic factors may have changed overtime. Lastly, having a garden, balcony or neither to sunbathe during social isolation measures may have had a significant impact on individual risks of vitamin D deficiency amidst the pandemic.

A multivariate analysis was done on our study population adjusting to potential confounders including sex, body mass index (BMI), frailty score, ethnicity, smoking status and comorbidities. We appreciate that socio-demographic factors measured by household income and Townsend deprivation index are important variables to consider. Nonetheless, this was difficult to determine in our study population who were over the age of 65 years with the majority being retired and receiving state pension. Comparable to our study, a systematic review and meta-analysis by Munshi and colleagues also identified that patients with poorer prognoses with COVID-19 was associated with lower serum levels of vitamin D [6]. A smaller retrospective observational study in Italy by Carpagnano et al. [7] identified a high prevalence of vitamin D deficiency in COVID-19 patients treated in intensive care units with hypovitaminosis associated with poorer prognostic outcomes of acute respiratory failure and mortality. 
Regardless, we appreciate that the evidence for vitamin $\mathrm{D}$ deficiency associated with severity of or mortality from COVID-19 infection remains sparse and a randomised controlled trial is needed to identify whether vitamin D supplementation will help prevent or treat COVID-19. It is also important to appreciate that a large proportion of patients with COVID-19 are either asymptomatic or have mild symptoms that do not require hospitalization. Whether vitamin D status and supplementation plays an important role in this cohort remains unclear.

Vitamin D has been identified to condition the innate and adaptive immune reaction to both bacterial and viral infections [3]. The COVID-19 pandemic as of 28 August 2020 has resulted in over 24 million people affected causing a devastating 827,246 deaths globally [8]. Whilst waiting for an effective vaccination and treatment for COVID-19, it would seem reasonable to encourage efforts to achieve reference nutritional intakes to optimise innate immune responses to infection. Vitamin D supplementation in accordance to national guidelines is a cheap intervention with a good safety profile that has multiple health benefits and should therefore be recommended to the general public.

Funding This research received no specific grant from any funding agency in the public, commercial or not-for-profit sectors. The authors do not have any financial disclosures or financial interests to declare.

\section{Compliance with ethical standards}

Conflict of interest All authors understand the policy of declaration of interests and all declare that we have no competing interests.

\section{References}

1. Hastie CE, Pell JP, Sattar N (2020) Vitamin D and COVID-19 infection and mortality in UK biobank. Eur J Nutr 1-4. https:// doi.org/10.1007/s00394-020-02372-4

2. NICE (2020) COVID-19 rapid evidence summary: vitamin D for COVID-19. https://www.nice.org.uk/advice/es28/evidence. Accessed 30 Aug 2020

3. Baktash V, Hosack T, Patel N et al (2020) Vitamin D status and outcomes for hospitalised older patients with COVID-19. Postgrad Med J 2020:138712. https://doi.org/10.1136/postgradmedj-2020138712

4. Waldron JL, Ashby HL, Cornes MP et al (2013) Vitamin D: a negative acute phase reactant. J Clin Pathol 66(7):620-622. https ://doi.org/10.1136/jclinpath-2012-201301

5. Ghashut RA, Talwar D, Kinsella J et al (2014) The effect of the systemic inflammatory response on plasma vitamin $25(\mathrm{OH}) \mathrm{D}$ concentrations adjusted for albumin. PLoS ONE 9(3):e92614. https://doi.org/10.1371/journal.pone.0092614

6. Munshi R, Hussein MH, Toraih EA et al (2020) Vitamin D insufficiency as a potential culprit in critical COVID-19 patients. J Med Virol. https://doi.org/10.1002/jmv.26360

7. Carpagnano GE, Lecce VD, Quaranta VN et al (2020) Vitamin D deficiency as a predictor of poor prognosis in patients with acute respiratory failure due to COVID-19. J Endocrinol Invest. https ://doi.org/10.1007/s40618-020-01370-x

8. WHO (2020) Weekly operational update of COVID-19 28 August 2020. https://www.who.int/docs/default-source/coronaviruse/situa tion-reports/wou-28-august-approved.pdf?sfvrsn=d9e49c20_2. Accessed 30 Aug 2020 